\title{
Tensile Characteristics of Low Density Infill Patterns for Mass Reduction of 3D Printed Polylactic Parts
}

\author{
M. N. F. Saniman ${ }^{*}$, M. H. M Hashim¹, K. A. Mohammad¹, K. A. Abd Wahid¹, W. M. Wan Muhamad¹ and N. H. Noor Mohamed² \\ ${ }^{1}$ Mechanical Engineering Section, Universiti Kuala Lumpur Malaysia France Institute, 43650 Bangi, Selangor, Malaysia \\ 2Department of Mechanical and Manufacturing Engineering, Universiti Malaysia Sarawak, 94300 Kota Samarahan, Sarawak.
}

\begin{abstract}
Various infill patterns are introduced in 3D printing to generate low density objects that leads to reduced cost and fabrication time through mass reduction. However, as a trade-off, the strength of the $3 \mathrm{D}$ printed component is uncertain. Confusions arise in determining the infill pattern with highest value of tensile strength since most studies limited only to rectilinear, honeycomb, and concentric infill patterns. As consequences, there are very little information on rarely used infill patterns such as Hilbert curve, Archimedean cord and octagram spiral. Therefore, the purpose of this research is to investigate and compare the tensile strength and strain of all infill patterns in mass reduction of 3D printed components experimentally. Following ASTM D638 type III standard, ten tensile test specimens of each infill patterns with $20 \%$ density were printed with an FFF 3D printer and were then tested. It was found that Archimedean cords infill pattern had the highest specific tensile strength of $33.23 \times 10^{3} \mathrm{MPa} \cdot \mathrm{mm}^{3} / \mathrm{g}$ which made it as the optimum infill pattern for the mass reduction of $3 \mathrm{D}$ printed parts with a high tensile strength. On the other hand, having the highest specific tensile strain of $18.21 \times 10^{3} \% \cdot \mathrm{mm}^{3} / \mathrm{g}$, concentric infill pattern was found to be more suitable for producing lightweight parts with a higher elongation before break. Additionally, Hilbert curve infill was the worst selection for mass reduction since it had the lowest values of specific tensile strength and specific strain of $19.80 \times 10^{3} \mathrm{MPa} \cdot \mathrm{mm}^{3} / \mathrm{g}$ and $8.34 \% \cdot \mathrm{mm}^{3} / \mathrm{g}$, respectively. Nevertheless, the trends of tensile strength and strain of all six infill patterns had been obtained, especially for rarely investigated infill patterns of Archimedean cords, octagram spiral, and Hilbert curve. Specifically, the trend from the strongest to the weakest (in \% compared to solid) for specific tensile strength is rectilinear (38.57\%), Archimedean chords (37.29\%), concentric (36.57\%), octagram spiral $(34.79 \%)$, honeycomb $(27.84 \%)$, and Hilbert curve $(22.25 \%)$, while for specific strain is concentric $(102.6 \%)$, octagram spiral (83.94\%), rectilinear (78.22\%), Archimedean cords $(77.99 \%)$, honeycomb $(54.84 \%)$, and Hilbert curve $(45.14 \%)$.
\end{abstract}

\author{
ARTICLE HISTORY \\ Revised: $24^{\text {th }}$ Mar 2020 \\ Accepted: $25^{\text {th }}$ Apr 2020 \\ KEYWORDS \\ 3D printing; \\ Mass reduction; \\ Infill pattern; \\ Tensile strength, Strain
}

\section{INTRODUCTION}

Fused Filament Fabrication (FFF) based three dimensional (3D) printing, which has been developed in 1980s, is a kind of manufacturing process to produce a three-dimensional products layer by layer [1]. For years, FFF 3D printers were used only by industries. The first at-home personal desktop 3D printer emerged in 2005, which allow individuals to experiment with various innovative product design as well as to create successful prototypes prior to actual manufacturing processes. For example, prototypes of hydrokinetic turbine blade [2], recirculation channel of turbocharger compressor [3], and Eddy current testing probe [4] could be produced via 3D printer based on the conducted numerical simulations and composed design in order to study it manufacturability and producibility [5]. It is due to the fact that 3D printing is generally faster, affordable and easier to use compared to other quick prototyping technologies. This technology also has increased the scholastic and industrial spirits of masses owing to its ability to create complex geometry with customizable material properties [6]. Since the outputs from 3D printers are highly customizable, users' interest in unique products may affect their likelihood to further adopt the current technology, which will only push the technology forward [7].

In product innovation, one of the purposes of using 3D printing technology is to reduce the amount of material used to fabricate any components. In consequences, not only the cost for production could be reduced, but also speeding up the overall manufacturing processes [8]. However, in order to produce the desired lightweight products, all related parameters in 3D printing must be considered such as the type of thermoplastic materials, structural parameters, 3D printing machine parameters, and build support parameters [9-11]. Sood, Ohdar and Mahaprata [12] investigated on layer thickness, printing orientation, raster angle, raster width and air gap while Moscato et. al. [13] studied the effect of various infill density to the mechanical and electrical properties of 3D printed materials. It is noted that there are more than one hundred printing parameters combined, which could affect the tensile strength of $3 \mathrm{D}$ printed parts. However, this study focused specifically on the infill patterns, since the selection of infill patterns affect directly with the parameters related to mass reduction such as weight and material usage. 